\title{
Non-chemotoxic induction of cancer cell death using magnetic nanowires
}

This article was published in the following Dove Press journal:

International Journal of Nanomedicine

17 March 2015

Number of times this article has been viewed

\author{
Maria F Contreras' \\ Rachid Sougrat ${ }^{2}$ \\ Amir Zaher ${ }^{3}$ \\ Timothy Ravasi ${ }^{1,3}$ \\ Jürgen Kosel ${ }^{3}$ \\ 'Division of Biological \\ and Environmental Sciences \\ and Engineering, ${ }^{2}$ Advanced \\ Nanofabrication Imaging \\ and Characterization, ${ }^{3}$ Division \\ of Computer, Electrical \\ and Mathematical Sciences \\ and Engineering, King Abdullah \\ University of Science and Technology, \\ Thuwal, Kingdom of Saudi Arabia
}

Video abstract

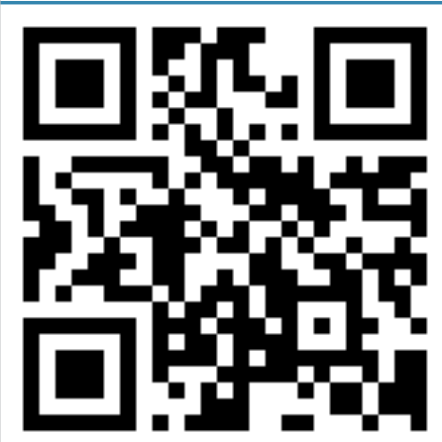

Point your SmartPhone at the code above. If you have a QR code reader the video abstract will appear. Or use:

http://dvpr.es/IFdlovh

Correspondence: Jürgen Kosel Electrical Engineering Department, 4700 King Abdullah University of Science and Technology, Thuwal 23955-6900,

Saudia Arabia

Tel +96654470056

Email jurgen.kosel@kaust.edu.sa
Abstract: In this paper, we show that magnetic nanowires with weak magnetic fields and low frequencies can induce cell death via a mechanism that does not involve heat production. We incubated colon cancer cells with two concentrations $(2.4$ and $12 \mu \mathrm{g} / \mathrm{mL})$ of nickel nanowires that were $35 \mathrm{~nm}$ in diameter and exposed the cells and nanowires to an alternating magnetic field $(0.5 \mathrm{mT}$ and $1 \mathrm{~Hz}$ or $1 \mathrm{kHz})$ for 10 or 30 minutes. This low-power field exerted a force on the magnetic nanowires, causing a mechanical disturbance to the cells. Transmission electron microscopy images showed that the nanostructures were internalized into the cells within 1 hour of incubation. Cell viability studies showed that the magnetic field and the nanowires separately had minor deleterious effects on the cells; however, when combined, the magnetic field and nanowires caused the cell viability values to drop by up to $39 \%$, depending on the strength of the magnetic field and the concentration of the nanowires. Cell membrane leakage experiments indicated membrane leakage of $20 \%$, suggesting that cell death mechanisms induced by the nanowires and magnetic field involve some cell membrane rupture. Results suggest that magnetic nanowires can kill cancer cells. The proposed process requires simple and low-cost equipment with exposure to only very weak magnetic fields for short time periods.

Keywords: cell death induction, low frequency alternating magnetic field, nanomedicine, nanowire internalization, nickel nanowires

\section{Introduction}

Micro- and nanostructured materials have become relevant to the life sciences mainly because manufacturing advances now permit their sizes to be tailored in a controlled fashion such that they match the sizes of biological entities, which can range from tens of nanometers (average size of a virus) to tens of micrometers (average size of a mammalian cell). ${ }^{1}$ Given the high surface area to volume ratio of such materials, the chances of a bond to be created or a payload to be delivered are dramatically increased with the use of nanostructured materials. Surface areas are commonly decorated with coatings that are intended for two purposes: to enhance the biocompatibility of the nanoparticle and to gain bioactivity, ie, to become an active player in the biological niche of interest. This bioactivity has been exploited for cell targeting, ${ }^{2,3}$ cargo payload (drug and gene delivery), ${ }^{4-6}$ contrast agents, ${ }^{7}$ or a combination of these, giving multifunctionality to a nanostructure. ${ }^{8-10}$

Magnetic nanoparticles (MNPs) can be remotely manipulated by magnetic fields. Using direct current fields, MNPs can be trapped, concentrated, ${ }^{11-14}$ or used in cell separation. ${ }^{15-17}$ Under alternating fields, MNPs can be heated ${ }^{18}$ or rotated, ${ }^{19,20}$ and, in cases of elongated structures, they can transmit forces or torques to whatever they are in contact with.

Investigation of magnetic nanostructures for biomedical applications has increased recently. Most previous studies utilized magnetic nanobeads, but recent studies have 
reported diverse bioapplications of magnetic nanowires (NWs) as well. NWs offer advantages over nanobeads, such as larger magnetic moments per unit of volume and larger surface area to volume ratios, which allow them to bind more efficiently to cells, producing purer populations during cell separation. $^{21,22}$

The two magnetic materials usually used as NWs in biomedical applications are Fe and Ni. Out of these two, Fe NWs tend to aggregate more, since they have a higher remanence magnetization value, ${ }^{23}$ making $\mathrm{Ni}$ a better material in that regard. Furthermore, Ni NWs have proven to be efficient in cell separation, manipulation, and purification ${ }^{16,17,21,22,24-26}$ as well as in delivering cargoes of various macro-particles including biological entities. ${ }^{27}$ Recent studies have investigated their use as therapeutic agents for hyperthermia ${ }^{28}$ and cell inflammation induction ${ }^{29}$ in cultures of human embryonic cells. They have also been shown to work as apoptotic agents for pancreatic cancer cells. ${ }^{30}$

The large number of studies that use Ni NWs in comparison to $\mathrm{Fe}$ might suggest that $\mathrm{Ni}$ is a better candidate material despite its reported genotoxicity and cytotoxicity in Ni-containing dust particles. ${ }^{31}$ Even though no work has been done that directly compares the cytotoxicity of $\mathrm{Fe}$ and Ni NWs under the same experimental conditions (cell line, incubation times, concentrations), a cross-comparison among studies $^{32,33}$ reveals that Fe NWs are considerably less toxic at a given concentration than are Ni NWs. However, as mentioned above, the stronger aggregation of Fe NWs limits the use of pristine NWs, requiring passivation to overcome this limitation.

In addition to work on biological and biomedical applications, fundamental studies involving internalization into different cell lines, ${ }^{34}$ the dependence of cytotoxicity on incubation time and NW concentration, ${ }^{33,34}$ and length-dependent cytotoxicity $^{35}$ have been reported. Moreover, cellular processes such as increased levels of reactive oxygen species, ${ }^{27}$ cell viability reduction, ${ }^{27,36}$ and cell membrane leakage ${ }^{36}$ have been shown to be activated solely by the incubation of cells with Ni NWs.

Alterations in cellular features due to magnetomechanical effects from alternating magnetic fields (AMFs) have been studied before. The first study applied a low-frequency $(1-10 \mathrm{~Hz})$, high-gradient magnetic field and mechanical vibration to mesenchymal stem cells and showed that both mechanisms play an important role in $\mathrm{F}$-actin remodeling and regulation of adipogenic differentiation. ${ }^{37}$ The next two studies ${ }^{38,39}$ incubated magnetic particles (iron oxide nanoparticles and permalloy microdisks, respectively) with cells in culture and applied AMFs. In the first one, ${ }^{38}$ a hyperthermia-like field was employed (16 mT, $260 \mathrm{kHz})$, while in the second one, ${ }^{39}$ a low-frequency field was used $(9 \mathrm{mT}, 20 \mathrm{~Hz})$. Both studies induced cell death in coincubated cells and particles after AMF exposure. Common changes in the cells included cell membrane leakage and cell shrinkage. The permalloy particles were functionalized with antibodies directed towards overexpressed cancer cell membrane receptors.

There have also been a few studies in which Ni NWs in combination with magnetic fields were used. Two studies have been conducted that used low-frequency magnetic fields to exploit the rotational response of $\mathrm{Ni} N W s{ }^{29,40}$ In the first one, Choi et $\mathrm{al}^{29}$ measured interleukin-6 (IL-6) expression (a common proinflammatory cytokine) after a 13 hour coincubation of cells with Ni NWs and application of a field (field strength not mentioned) for 5 minutes. The IL-6 expression fold-changes (compared to cells incubated with NWs only, no applied field) were: 5, 4.5, and 1.5 for 1.7 Hz, $8.3 \mathrm{~Hz}$, and $11.6 \mathrm{~Hz}$, respectively. The second study consisted of the addition of Ni NWs to mouse fibroblasts in culture and the subsequent application of a very strong field (240 mT, $1 \mathrm{~Hz}$ ) for 20 minutes. Fung et al showed an 89\% cell viability reduction. ${ }^{40}$ Moreover, the NWs by themselves did not induce elevated expression of IL-6 when cocultured with mouse fibroblasts for 12 hours, despite the moderate cytotoxicity reported for $\mathrm{Ni}^{41}$ and the strong hypersensitivity of human skin. ${ }^{42}$

The studies mentioned here used NWs with diameters ranging from 150 to $280 \mathrm{~nm}$. For assessing cell damage, optical microscopy and quantitative real-time polymerase chain reaction (PCR) were used. Only Fung et $\mathrm{al}^{40}$ used a colorimetric method to compare viability of a given cell population before and after application of the low-frequency AMF.

In the present study, we applied a low-frequency, smallamplitude AMF to cells that had been incubated with $\mathrm{Ni}$ NWs. Compared with previous studies, the applied magnetic field was much weaker in our experimental setup and the NWs were about one order of magnitude thinner than those used in previous work. The motivation behind this experimental setup is the exploitation of a non-chemotoxic way of inducing cancer cell death.

Here, we consider NW-cell interactions by using transmission electron microscopy (TEM) images that reveal the NW internalization process and the location of the magnetic NWs inside cancer cells. We also consider AMF-cell interactions when the magnetic field is applied for short periods of time (10 or 30 minutes) to cells in culture. Finally, we discuss NW-AMF interactions and estimate the forces exerted by 
a single NW, along with the combined effects of NW-cell interaction in the presence of an AMF, which we call AMF treatment. We report on cell population assays that measured viability and membrane leakage.

\section{Materials and methods Fabrication of magnetic NWs}

The NWs were fabricated by electrodeposition into alumina membranes. Full details of the aluminum anodization $^{43}$ and pulsed electrodeposition processes can be found elsewhere. ${ }^{44,45}$ Briefly, a highly pure aluminum substrate (99.999\%; Goodfellow, London, UK) was cleaned and electropolished. A two-step anodization of the polished aluminum under specific conditions $\left(0.3 \mathrm{M}\right.$ oxalic acid, $\left.4^{\circ} \mathrm{C}, 40 \mathrm{~V}\right)$ resulted in the growth of a porous anodic alumina template with hexagonally arranged nanopores with diameters from 30 to $40 \mathrm{~nm}$, which was used as the template for NW fabrication. Pulsed electrodeposition was employed to deposit $\mathrm{Ni}$ with current pulses limited to $30 \mathrm{~mA}$. The NW length was controlled by the deposition time, and $4 \mu \mathrm{m}$ long Ni NWs $(4.1 \pm 1.4 \mu \mathrm{m})$ were fabricated.

Afterwards, the template containing the NWs was dissolved with $1 \mathrm{M} \mathrm{NaOH}$. The NWs were collected with a magnetic rack (DynaMag ${ }^{\mathrm{TM}}$-2; Life Technologies, Carlsbad, CA, USA), rinsed thoroughly with ethanol with 5 -second sonication steps in between the cleaning steps, and resuspended in cell culture medium. The cell culture medium was changed three times to remove any remaining ethanol.

\section{Characterization of magnetic NWs}

The NW morphology and geometrical features were investigated with scanning electron microscopy (SEM) and TEM (SEM: Quanta 3D; FEI Company, Hillsboro, OR, USA; and TEM: Tecnai BioTWIN; FEI Company). The length and diameter distributions were obtained directly from SEM images using ImageJ software. The NW composition was measured using energy-dispersive X-ray spectroscopy (scanning TEM Tecnai BioTWIN; FEI Company). All electron microscopic images were taken of NWs freshly released from the template after rinsing several times with ethanol. Once released, the NWs were sonicated for 10 minutes to achieve a better dispersion.

The NWs were quantified via an indirect method. After electrodeposition, the samples were briefly immersed in a chromium-based solution to partly reveal the NWs. After washing with ethanol, they were imaged with SEM, and the pore filling was found to be above $95 \%$. The number of pores of a certain area, quantified from an SEM image, was extrapolated from the total deposition area determined by the experimental setup. Due to the high pore filling, the total number of NWs was considered to be equal to the total number of pores.

Magnetization loops of NWs inside the alumina membrane were measured at room temperature with a vibrating sample magnetometer (VSM model MicroMagTM 3900; Princeton Measurement Corporation, Westerville, $\mathrm{OH}, \mathrm{USA}$ ) (maximum field used: $1 \mathrm{~T}$; sensitivity: $0.5 \mu \mathrm{emu}$; standard deviation at 1 second per measured point).

The surface charge of Ni NWs was measured by dynamic light scattering (Zetasizer Nano ZS, He-Ne laser 633 nm; Malvern Instruments, Malvern, UK). A concentrated solution of NWs was resuspended in complete culture medium, put into a capillary cell, and measured.

\section{Cell culture}

HCT116 (ATCC ${ }^{\circ}$ CCL-247 ${ }^{\mathrm{TM}}$ ) colorectal carcinoma cells were cultured following vendor recommendations in McCoy's 5A medium supplemented with $10 \%$ fetal bovine serum and $1 \%$ penicillin/streptomycin (final concentration). The doubling time of the cells in culture was found to be 17 hours.

\section{AMF treatment}

Cells were seeded in 96 -well plates $\left(5 \times 10^{4}\right.$ cells $/$ well $)$. After a 24-hour growth period and a confluence of about $80 \%$, NWs were added at concentrations of $2.4 \mu \mathrm{g} / \mathrm{mL}$ or $12 \mu \mathrm{g} / \mathrm{mL}$. The NWs were incubated with the cells for 1 hour before the magnetic field was applied for 10 or 30 minutes. Each experiment included negative controls (no NWs added and no field applied), a field-only control, and a NW-only control. Each experiment was carried out on three independent biological replicas. The AMF was produced using an in-house-made four-layer coil with six turns per layer of Litz wire in combination with a bipolar amplifier (AL-200-HF-A; Amp-Line Corporation, West Nyack, NY, USA) and a signal generator (33250A; Agilent Technologies, Santa Clara, CA, USA). The field amplitude was $0.5 \mathrm{mT}$ and the frequencies tested were $1 \mathrm{~Hz}$ and $1 \mathrm{kHz}$. The power required to generate this magnetic field corresponded to about $50 \mathrm{~mW}$.

\section{Cytotoxicity assays}

The effects of the different experiments on the cell viability were evaluated using two methods based on cell toxicity: a colorimetric assay that measures the reduction of the yellow compound, 3-(4,5-dimethylthiazol-2yl)-2,5-diphenyl tetrazolium bromide (MTT), to purple formazan by mitochondrial 
enzymes in active cells; ${ }^{46}$ and a fluorescent cell membrane integrity assay that measures the amount of lactate dehydrogenase (LDH), normally present inside cells, in the cell culture medium. The experimental details for each of the assays used are described below.

Immediately after AMF treatment, the cells were incubated with $100 \mu \mathrm{L}$ of $10 \%(\mathrm{v} / \mathrm{v})$ MTT solution $(0.5 \mathrm{mg} / \mathrm{mL}$ in phosphate-buffered saline) in cell culture medium for 2 hours. The MTT solution was carefully removed and the cells were lysed by adding $100 \mu \mathrm{L}$ of lysis solution $(20 \%$ [wt/v] sodium dodecyl sulfate $+0.6 \%[\mathrm{v} / \mathrm{v}] 37 \% \mathrm{HCl}$ in dimethyl sulfoxide). ${ }^{47}$ The MTT formazan crystals were dissolved by gently tapping the plate, and their optical density (OD) was measured by a microplate reader $\left(\mathrm{xMark}^{\mathrm{TM}}\right.$ microplate-absorbance-spectrophotometer; Bio-Rad Laboratories Inc., Hercules, CA, USA) at a test wavelength of 570 $\mathrm{nm}$ and a reference wavelength of $630 \mathrm{~nm}$. The cell viability was calculated as the percentage of optical density of the treated cells compared with the negative control (NC), ie, the untreated cells:

$$
\text { Viability } \%=\frac{O D[\text { Treated cells }]}{O D[N C \text { cells }]} \times 100 .
$$

For the membrane integrity assay, HCT 116 cells were seeded in medium supplemented with $5 \%$ fetal bovine serum, as the $10 \%$ concentration led to a higher background (BKGD) fluorescence. Additional controls were seeded for each experiment: BKGD wells (only 5\% cell culture medium), positive control (PC; lysed untreated cells to get a maximum cell membrane leakage value), and apoptotic cells (untreated cells exposed for 1 hour to ultraviolet light). After AMF treatment, 96-well plates were equilibrated to room temperature for 30 minutes. After this, $2 \mu \mathrm{L}$ of lysis solution $(9 \%[\mathrm{w} / \mathrm{v}]$ Triton $^{\circledR}$ X-100 in water) were added to the PC wells. Then, $120 \mu \mathrm{L}$ of Cytotox-ONETM reagent was added to each well to initiate the reaction that would lead to the production of the fluorescent product (resorufin) proportional to the amount of LDH in the medium. After 10 minutes at room temperature, the reaction was stopped by addition of $50 \mu \mathrm{L}$ of stop solution. The fluorescence signal was collected with the GloMax Multi Detection System (Promega Corporation, Fitchburg, WI, USA), using a green fluorescent filter (excitation $525 \mathrm{~nm}$, emission 580-640 nm). The percentage of LDH leakage was calculated as the average fluorescence values of the treated cells minus the average BKGD fluorescence signal normalized to the $\mathrm{PC}$, ie, the maximum membrane leakage less the average BKGD fluorescence signal:

$$
L D H \text { leak } \%=\frac{\text { Treated cells }-B K G D}{P C \text { cells }-B K G D} \times 100 .
$$

\section{Internalization of magnetic NWs}

Colon cancer cells were seeded in six-well plates until reaching a confluency of $80 \%$. Ni NWs were added in independent wells at a NW concentration of $2.4 \mu \mathrm{g} / \mathrm{mL}$ for 1 hour. Then, the medium was gently aspirated and the cells were fixed with $2 \mathrm{~mL}$ of $2.5 \%(\mathrm{v} / \mathrm{v})$ glutaraldehyde in cacodylate buffer (0.1 M, pH 7.4) for a minimum of 48 hours. The cells were kept at $4{ }^{\circ} \mathrm{C}$ until further processing. Fixed cells were treated with reduced osmium (1:1 mixture of $2 \%$ aqueous potassium ferrocyanide) as described previously, ${ }^{48}$ dehydrated in ethanol and embedded in epoxy resin. One hundred to $150 \mathrm{~nm}$ thick sections were collected on copper grids and stained with lead citrate.

Imaging was performed using a transmission electron microscope operating at $300 \mathrm{kV}$ (Titan Cryo Twin; FEI Company). The images were recorded by a $4 \mathrm{k} \times 4 \mathrm{k} C \mathrm{CD}$ camera (Gatan Inc., Pleasanton, CA, USA).

\section{Statistical analysis}

The data are expressed as means \pm standard deviation. The statistical comparisons of means were performed using oneway analysis of variance. The differences were considered to be significant for $P$-values of less than 0.05 .

\section{Results and discussion Characterization of magnetic NWs}

Figures $1 \mathrm{~A}$ and B show SEM and TEM images of Ni NWs, respectively. In both cases, dendrites, a feature resulting from the fabrication process, are distinguishable at one end of the NWs. The inset in Figure 1B shows in detail a NW section in which an outer layer of $6.0 \pm 1.2 \mathrm{~nm}$ thickness can be observed. Its composition was corroborated using single point energy-dispersive X-ray spectroscopy. Spectra of Figures $1 \mathrm{C}$ and D show the composition of the core and outer shell, respectively. The same materials are found in both cases but in different proportions; the core is predominantly composed of $\mathrm{Ni}$ as expected whereas the outer later contains mainly oxygen and carbon (the latter most likely comes from the ethanol in which NWs are stored). This oxide layer forms during the exposure of the NWs to air, $\mathrm{NaOH}$ (for the dissolution of the alumina), and ethanol cleaning steps.

Figure 2 shows the magnetization loops of an array of NWs embedded in the alumina oxide template. Array values of saturation magnetization $\left(M_{\mathrm{S}}\right)$ and coercive field $\left(H_{\mathrm{C}}\right)$ for the field that was applied parallel to the NWs are $46.7 \mathrm{Am}^{2} / \mathrm{kg}$ 

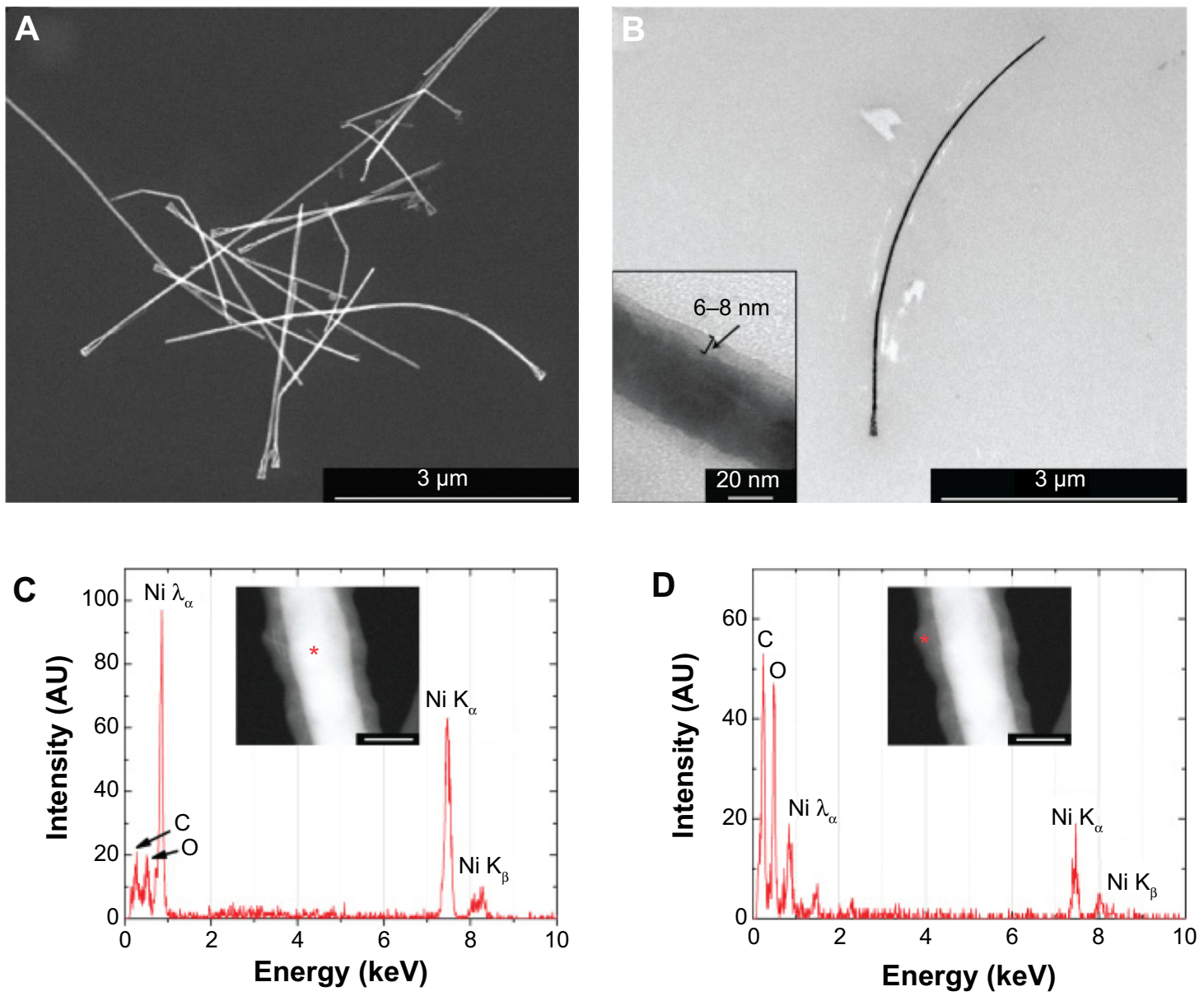

Figure I Morphology and composition analysis of magnetic nonowires.

Notes: (A) SEM image of Ni NWs on top of a silicon wafer substrate. (B) TEM image of a single Ni NW. The inset shows the outer oxide layer that is 6-8 nm thick. (C) Point EDX spectra of a Ni NW core and (D) its surrounding layer. The insets show the corresponding STEM images. Red asterisks indicate the point from which the spectrum was measured. Scale bars: $40 \mathrm{~nm}$.

Abbreviations: NW, nanowire; SEM, scanning electron microscopy; STEM, scanning transmission electron microscopy; TEM, transmission electron microscopy.

and $79 \mathrm{mT}$, respectively. The $M_{\mathrm{S}}$ value is smaller than the $M_{\mathrm{S}}$ values reported for bulk $\mathrm{Ni}$ in the literature $\left(54.3 \mathrm{Am}^{2} / \mathrm{kg}\right){ }^{49}$ We attributed this to the surface oxidation of the NWs.

It is important to note that the magnetization loop of a single NW deviates considerably from the one of an array, in which NWs interact magnetostatically with each other. Single Ni NWs show single domain properties. Therefore, they are permanently magnetized with the $M_{\mathrm{R}}$ value being equivalent to the one of $M_{\mathrm{S}}$ measured in the parallel direction. ${ }^{50}$

\section{Magnetic NWs-cells interaction}

Once the NWs are in close proximity to the cellular membrane, an uptake mechanism is triggered that in turn determines the intracellular fate of the nanostructures. Endocytosis has been accepted as the most common passive mechanism for MNP uptake by different cellular types (HeLa, fibroblast, and dendritic cells). ${ }^{38}$ Also, uptake and intracellular location of nanostructures have been shown to depend on several factors such as the NW aspect ratio, cell line, NW surface charge, etc. ${ }^{51}$

The zeta potential measured for the Ni NWs was found to be $-15.1 \mathrm{mV}$. Such a value is considered low, ${ }^{52}$ yielding only a small electrostatic repelling force. The NWs tend to aggregate, which is in agreement with the observations made for released NWs.

Negatively charged particles bind less efficiently to cell surfaces compared with neutral or positively charged particles..$^{53}$ The efficacy of internalization is improved in positively charges particles. ${ }^{54}$ In the case of Ni NWs, the surface charge (almost neutral) is favorable to internalization efficacy, which under the conditions tested here was rather high, given that some NWs were found to be fully internalized after only 1 hour of incubation. Similar results were previously reported for thick Ni NWs (200 nm diameter) that were observed to cross the cell membrane after 40 minutes of incubation with rat neuroblastoma cells ${ }^{17}$ and for thin 


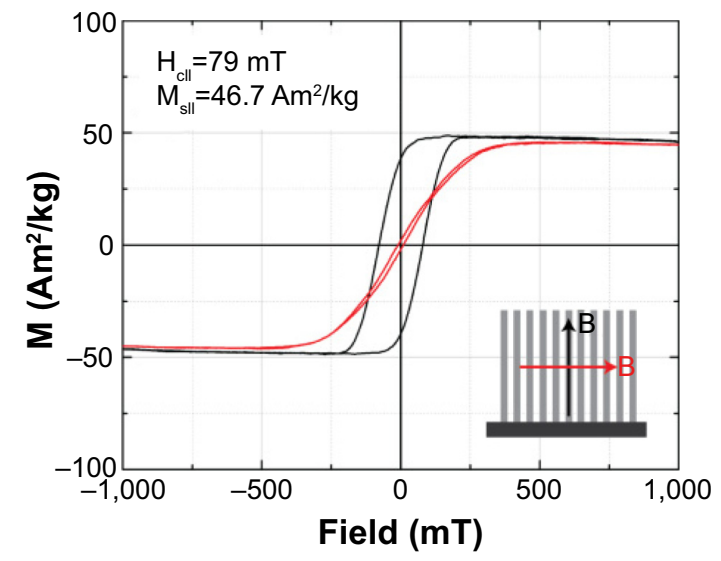

Figure 2 Magnetization loops of an array of Ni NWs embedded in the alumina membrane with magnetic field applied in the in-plane and out-of-plane directions. Notes: Black: in-plane direction. Red: out-of-plane direction. $\mathrm{H}_{\mathrm{c} \|}$ and and $\mathrm{M}_{\mathrm{s} \|}$, refer to the coercive field and the saturation when the field is applied in the in-plane direction (black) of the nanowires.

Abbreviations: M, magnetization; NWs, nanowires.

magnetic NWs ( $50 \mathrm{~nm}$ diameter) that were fully internalized after 2 hours of incubation with HeLa cells. ${ }^{32}$

To analyze the uptake and intracellular fate of Ni NWs, colon cancer cells incubated with Ni NWs were fixed, carefully sliced, and imaged by TEM. Two cell samples were prepared and imaged in replicate: a control sample (no NWs added) and a sample of cells incubated with Ni NWs.

Figure 3A shows four cells to which no NWs were added. Typical cell compartments and organelles are clearly identified, such as the nucleus, mitochondria, Golgi apparatus (GA), and the plasma membrane. Moreover, additional features include dark-colored vesicles that correspond to lipid droplets. Lipid droplets and bilayer membranes (nuclear, cellular, and from organelles like mitochondria and GA) can be easily distinguished, as they are darker. This is because the osmium stain used in the sample preparation tints glycoproteins and lipids. A final feature is the polarity observed in the cells. Colon cancer cells are epithelial cells with a polarity that is characterized by cilia-like formations in their apical membrane (bottom-right corner of Figure 3A).

Figure 3B shows NWs attached to the cell surface, particularly to the cilia-like structures from the apical membrane. Figures $\mathrm{C}$ and $\mathrm{D}$ show the cell membrane invagination process that starts from contact areas between the cell and NWs. The inset in Figure 3C shows in detail the formation process of a vesicle. Red arrows in both insets of Figure 3D show the cell membrane surrounding two NWs, indicating the invagination or engulfment process. Finally, Figures $3 \mathrm{E}$ and $\mathrm{F}$ show fully internalized NWs. Figure 3E shows a group of NWs completely contained inside a vesicle and Figure $3 \mathrm{~F}$ shows a large aggregation of NWs again completely internalized.
Some organelle reorganization is seen and is highlighted with the red arrow pointing at the GA that changed shape.

The images in Figure 3 show that within 1 hour of incubation time, all internalization steps (binding, invagination, and full internalization) were completed. It is very likely that the aggregates observed in some cases were already formed before being internalized by the cell. However, considering the micron-scale sizes of these aggregates (Figure 3F), the internalization of such structures was considerably fast. This is not entirely a surprise, since it has been shown that cells in culture can uptake micron-sized structures, the largest being a $3 \mu \mathrm{m}$ diameter sphere made of a cobalt-copper alloy ${ }^{55}$ and the longest a $20 \mu \mathrm{m}$-long Ni NW.

Figure 4 shows the dependence of cell viability on the NW concentration using the MTT assay. A concentration of $2.4 \mu \mathrm{g} / \mathrm{mL}$ did not significantly affect cell viability, whereas a significant difference $(P<0.01)$ was measured for $12 \mu \mathrm{g} / \mathrm{mL}$, which caused a slightly less than $90 \%$ drop in viability. The results suggest that NWs at the given concentrations do not induce major cytotoxic effects on cells. However, our experiments were carried out for an incubation time of only 1 hour. The cytotoxic effects might increase with longer incubation times, as has been shown before. ${ }^{33,36}$ This would have to be taken into account in in vivo applications. For instance, it has been shown that iron oxide nanoparticles remain in an organism for several days ${ }^{56}$ or even up to 6 months. ${ }^{57}$

\section{AMF-cells interaction}

Figure 5 shows MTT results when an AMF was applied to colon cancer cells using the MTT assay. When the AMF was applied for 10 minutes, the viability did not change with both field frequencies. When the AMF was applied for 30 minutes, the viability was lower but significantly different $(P<0.05)$ only for the $1 \mathrm{kHz}$ frequency, for which the viability dropped to $95 \%$.

The influence of similar AMFs on living organisms has been reported to be detrimental to bacteria, ${ }^{58-60}$ favorable to cancer cells ${ }^{55}$ and neutral to mammalian cells. ${ }^{61,62}$ Exposing bacteria (Escherichia coli and Staphylococcus aureus) to fields about ten-times higher in strength than in our experiments $(50 \mathrm{~Hz}, 10 \mathrm{mT})$ for 10 minutes slightly negatively affected the colony forming unit density. ${ }^{58}$ Another study on three mammalian cell lines found that cell proliferation increased by $30 \%$ for all cell lines after applying an AMF $(1 \mathrm{mT}$ and $50 \mathrm{~Hz}$ ) for 72 hours accompanied by DNA damage. ${ }^{62}$ Even though the field parameters in these earlier studies were close to the ones used in our experiments, the reason for different results (without considering variations 

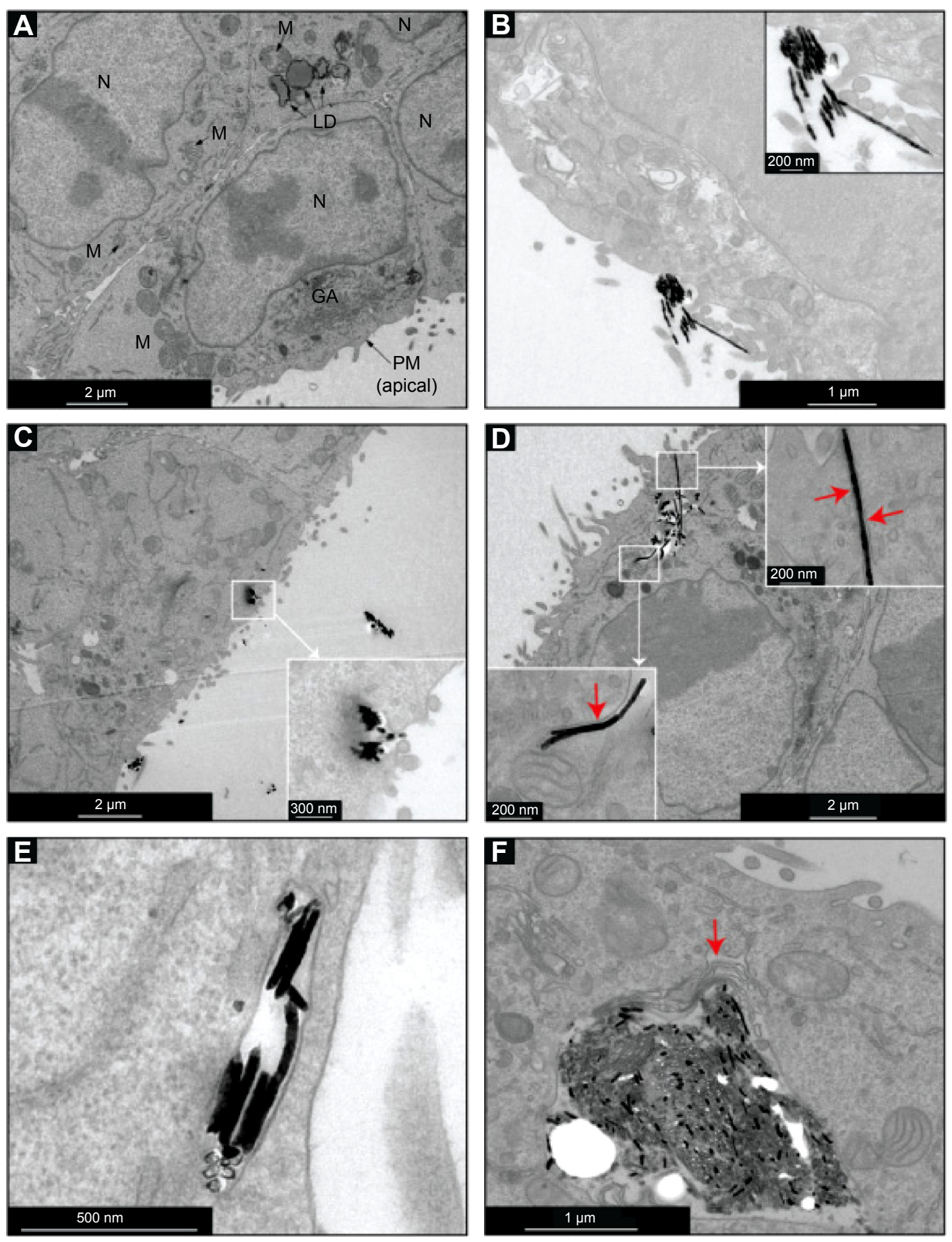

Figure 3 TEM images of colon cancer cells incubated for I hour with Ni NWs.

Notes: (A) Control cells (cells to which no NWs were added) with organelles such as M, GA, N, PM, and LD. (B) NWs in close proximity to the PM of colon cancer cells. Cells that internalized NWs as aggregates (C) and as single NWs (D). PM surrounding indivdual NWs are visible (red arrows). (E and F) Fully internalized NWs. A few NWs can be observed fully surrounded by a membrane vesicle $(E)$ and a fully internalized large aggregate of NWs caused the reordering of organelles like the GA (red arrow) (F).

Abbreviations: GA, Golgi apparatus; LD, lipid droplets; M, mitochondria; N, nucleus; NW, nanowire; PM, plasma membrane; TEM, transmission electron microscopy.

inherent to the experimental setup and specific cell-line responses) might come from the long times the cells were exposed to fields $(24,48 \text {, and } 72 \text { hours })^{62}$ compared with 10 or 30 minutes in this study.

\section{AMF treatment}

The behavior of a magnetic NW in the presence of an AMF is governed by its magnetization. In the case of NWs made of $\mathrm{Ni}$, the orientation of the magnetization is determined 


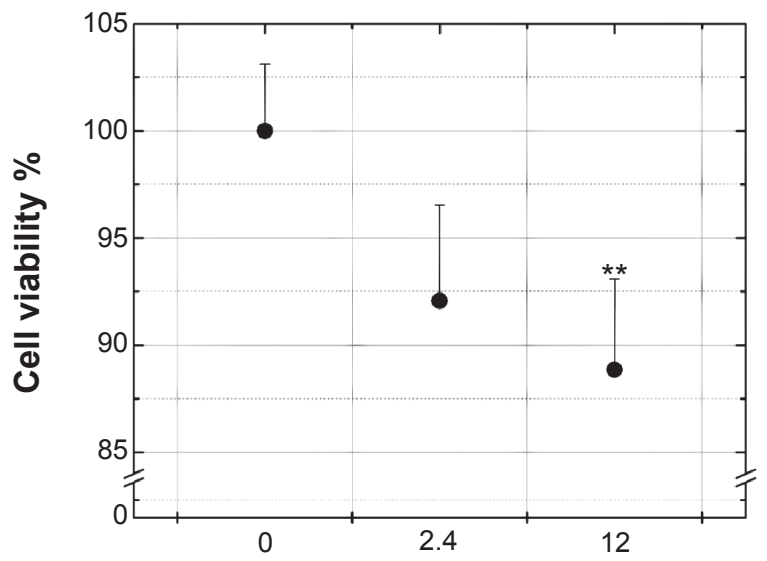

Ni NW concentration $(\mu \mathrm{g} / \mathrm{mL})$

Figure 4 Viability of colon cancer (MTT assay) cells after I hour incubation with Ni NWs.

Notes: The NC sample ( $0 \mathrm{Ni} \mu \mathrm{g} / \mathrm{mL})$ corresponds to cells without $\mathrm{NW}$ addition. $\mathrm{NW}$ concentration values are expressed and $\mathrm{Ni} \mu \mathrm{g}$ per $\mathrm{mL}$ of cell culture medium. Data represents means \pm standard deviation, $n=3, * * p<0.0$ I versus $N C$.

Abbreviations: MTT, 3-(4,5-dimethylthiazol-2yl)-2,5-diphenyl tetrazolium bromide; NC, negative control; NW, nanowire.

by the strong shape anisotropy, which dominates the weak magnetocrystalline anisotropy, and the magnetic easy axis, which lies along the NW axis. ${ }^{23}$ Therefore, when the NWs are exposed to an AMF, they will experience torque while trying to align the magnetic moment with the field.

The continuous realignment with the AMF was experimentally visualized with an inverted microscope (Nikon Eclipse $\mathrm{T} i$ ) of an agglomeration of released Ni NWs resuspended in cell culture medium with an AMF of $0.5 \mathrm{mT}$ and various frequencies $(0.1,1$, and $10 \mathrm{~Hz}$; Video S1, S2, and $\underline{\mathrm{S} 3}$, respectively).

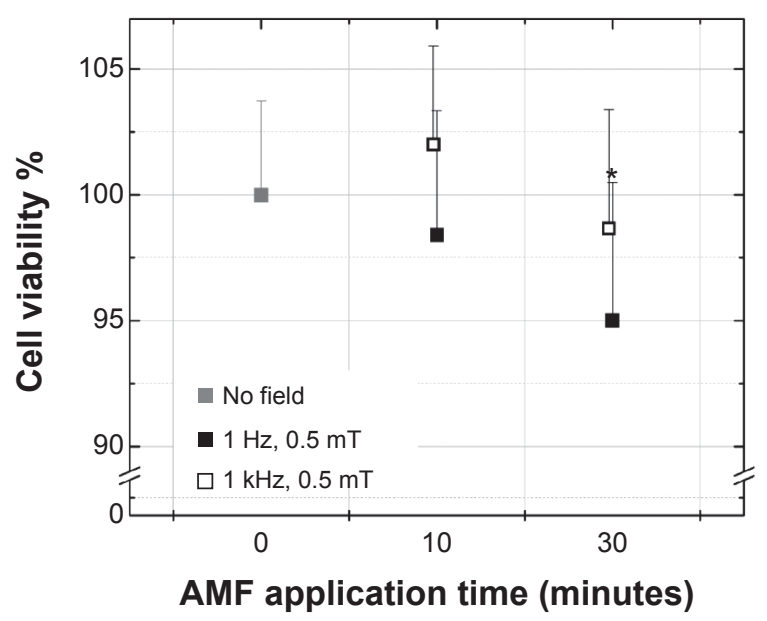

Figure 5 Viability of colon cancer cells (MTT assay) after exposure to AMFs of two different frequencies.

Notes: The negative control sample ( 0 minutes of AMF) corresponds to cells without AMF exposure. Data represent means \pm standard deviation, $n=3, * P<0.05$. Abbreviations: AMF, alternating magnetic field; MTT, 3-(4,5-dimethylthiazol-2yl)2,5-diphenyl tetrazolium bromide.
The magnetic torque, $\tau_{m}$, exerted by the field on a single NW is given by:

$$
\tau_{m}=m \times B=\mu_{0} m \times H=\mu_{0} m H \sin \theta
$$

where $m$ is the magnetic moment, $B$ the magnetic field, $\mu_{0}$ the permeability of free space, $H$ the magnetic field strength, and $\theta$ the angle between $m$ and $H$. Considering $m=M V$ ( $M$ is the magnetization and $V$ the volume), $V=\pi r^{2} l$ (with $r$ the NW radius and $l$ its length), and $H=H_{0} \sin (\omega t)$, equation 3 can be rewritten as:

$$
\tau_{m}=\mu_{0} M_{R} \pi r^{2} l H_{0} \sin (\omega t) \sin \theta
$$

In equation 4, the value of $M_{R}$ replaces $M$, which for a single NW can be calculated from the value of $M_{S}$ measured for the array. This value is obtained from Figure 2, and together with the estimation of the total number of NWs in the measured piece (determined as described in the Materials and methods section), $M_{R}$ of a single NW is $47.4 \mathrm{Am}^{2} / \mathrm{kg}$. The maximum value of $\tau_{m}$ is obtained for $\theta=90^{\circ}$ and it corresponds to $0.81 \times 10^{-18} \mathrm{~N} \cdot \mathrm{m}$. From this value, the force a NW exerts on a cell in the presence of an AMF can be calculated using the simple model illustrated in Figure 6.

If we assume that the NW rotates about its midpoint, the magnitude of the force acting on each of its ends is $0.2 \mathrm{pN}$. This value is well below the force required to disrupt the membrane, which is on the order of $100 \mathrm{pN} .{ }^{63}$ However, there are other mechanisms affecting the cells that rely on much weaker forces. ${ }^{64}$ Forces of a few $\mathrm{pN}$ can cause changes

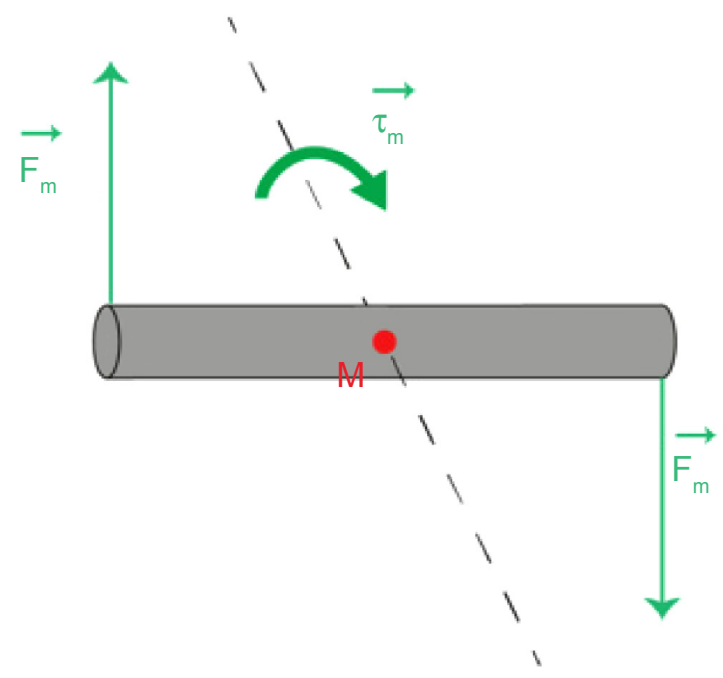

Figure 6 A simple model for estimating the force an AMF exerts on a NW that can be transmitted to a cell if the NW's ends are attached to it.

Abbreviations: $\tau_{m}$, magnetic torque; AMF, alternating magnetic field; $F_{m}$, magnetic force; $M$, midpoint; $N W$, nanowire. 
in protein conformation ${ }^{65}$ and clustering of membraneassociated molecules ${ }^{66}$ that could lead to the activation of various signaling pathways influencing cellular behavior and fitness. Such processes might be activated when the AMF treatment is applied and can be partly responsible for the measured reduction in cell viability. AMF treatment significantly reduces the cell viability (Figure 7), which drops to about $78 \%$ in the case of a concentration of $2.4 \mu \mathrm{g} / \mathrm{mL}$, and to between $60 \%$ and $70 \%$ in the case of $12 \mu \mathrm{g} / \mathrm{mL}$. For the $2.4 \mu \mathrm{g} / \mathrm{mL}$ concentration, the frequency does not influence the cell viability values, whereas at $12 \mu \mathrm{g} / \mathrm{mL}$, the viability is slightly more affected at higher frequencies, yielding a drop of $38 \%$.

During the AMF treatment, the temperature was monitored and a maximum difference of $1.9^{\circ} \mathrm{C}$ was measured with respect to the control cells. Such small temperature changes have been shown to slightly affect cell numbers with incubation times of 1 hour. ${ }^{67}$ Since the cells in our experiments were exposed to the temperature change for only 10 minutes, we attribute the reduction in cell viability to the magnetic actuation of the NWs. This is in agreement with the observed independence of frequency from viability, since the force exerted by the NWs on the cells was independent of the frequency (as long as the dynamic responses of the NWs could follow the field). Even though there was a five-fold difference in NW concentrations between the two concentrations tested, the drop in cell viability did not increase by a factor of more than two. A possible explanation for this was NW aggregation, which led to a

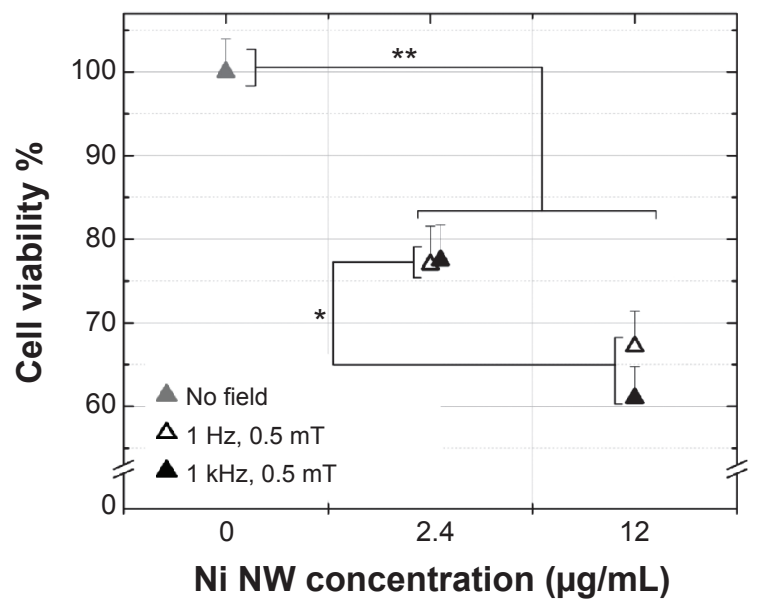

Figure 7 Cell viability of colon cancer (MTT assay) cells incubated with Ni NWs for I hour and then exposed to magnetic fields of different frequencies and amplitudes for 10 minutes.

Notes: In the NC cells ( $0 \mathrm{Ni} \mu \mathrm{g} / \mathrm{mL})$, no NWs were added (I $00 \%$ cell viability value). Data represent means \pm standard deviation, $n=3, * P<0.05 ; * * p<0.01$ versus NC. Abbreviations: MTT, 3-(4,5-dimethylthiazol-2yl)-2,5-diphenyl tetrazolium bromide; NC, negative control; NW, nanowire. nonuniform distribution of the NWs, when added to cells, and which became more evident when the NW concentration was increased.

Figure 8 shows the cell membrane leakage when the cells with the two NW concentrations underwent AMF treatment. The cells exhibited LDH leakage between $32 \%$ and $36 \%$, which turns out to be significantly different from the leakage from negative control cells and cells in which apoptosis was induced. While the calculations above indicated that the force exerted by a NW on the cell membrane was not large enough to result in a rupture of the cell membrane, the AMF treatment did affect the integrity of the cell membrane. We attribute this to two effects. The first is that the effect of the combined forces of several single NWs acting on a membrane can be considerably larger than the effect of force of a single NW. For such an effect to occur is highly likely considering that the NW concentrations were equivalent to $100 \mathrm{NWs} /$ cell and $500 \mathrm{NWs}$ /cell (Table S1). The second effect is from NW agglomerations, which we frequently observed during the experiments, from which the generated forces can be many times higher than the force of a single NW. Membrane leakage was also evaluated for cells incubated with only NWs and cells in which only AMF was applied for 10 minutes (Figure S1). The NWs and the AMFs turned out to influence membrane integrity to the same degree (leakage values were between $18 \%$ and $23 \%$ ).

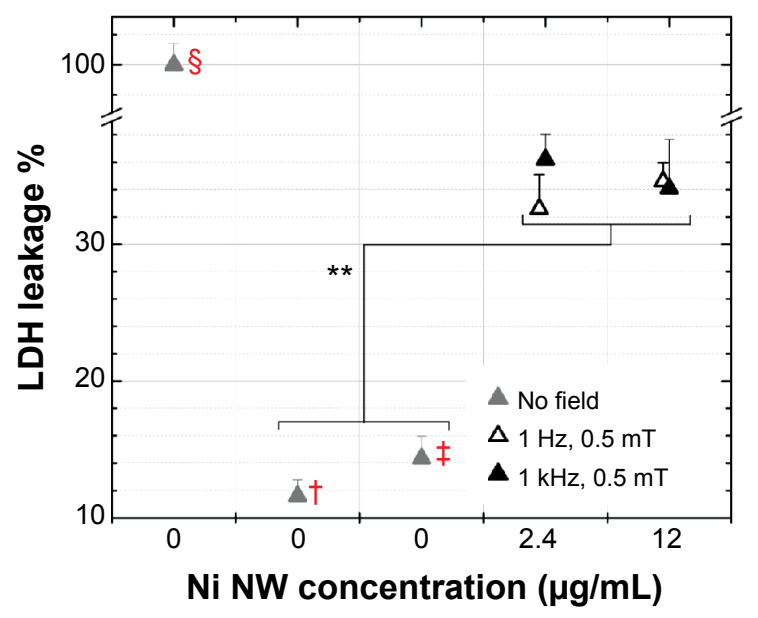

Figure 8 LDH leakage of colon cancer cells incubated with Ni NWs for I hour and then exposed to magnetic fields of different frequencies and amplitudes for 10 minutes.

Notes: There are three control samples in which no NWs or fields were added (gray triangles). A sample of lysed cells $(\boldsymbol{\S})$ was used as the PC, which corresponded to $100 \%$ leakage. No NWs were added to the NC ( $\dagger$ ) cells. Apoptosis was induced to cells $(\ddagger)$ by exposing them to ultraviolet radiation for I hour. Data represent means \pm standard deviation, $n=3, * * P<0.01$ versus NC. $P<0.01$ for $P C$ versus all the other samples (data not shown).

Abbreviations: LDH, lactate dehydrogenase; NC, negative control; NW, nanowire; PC, positive control. 


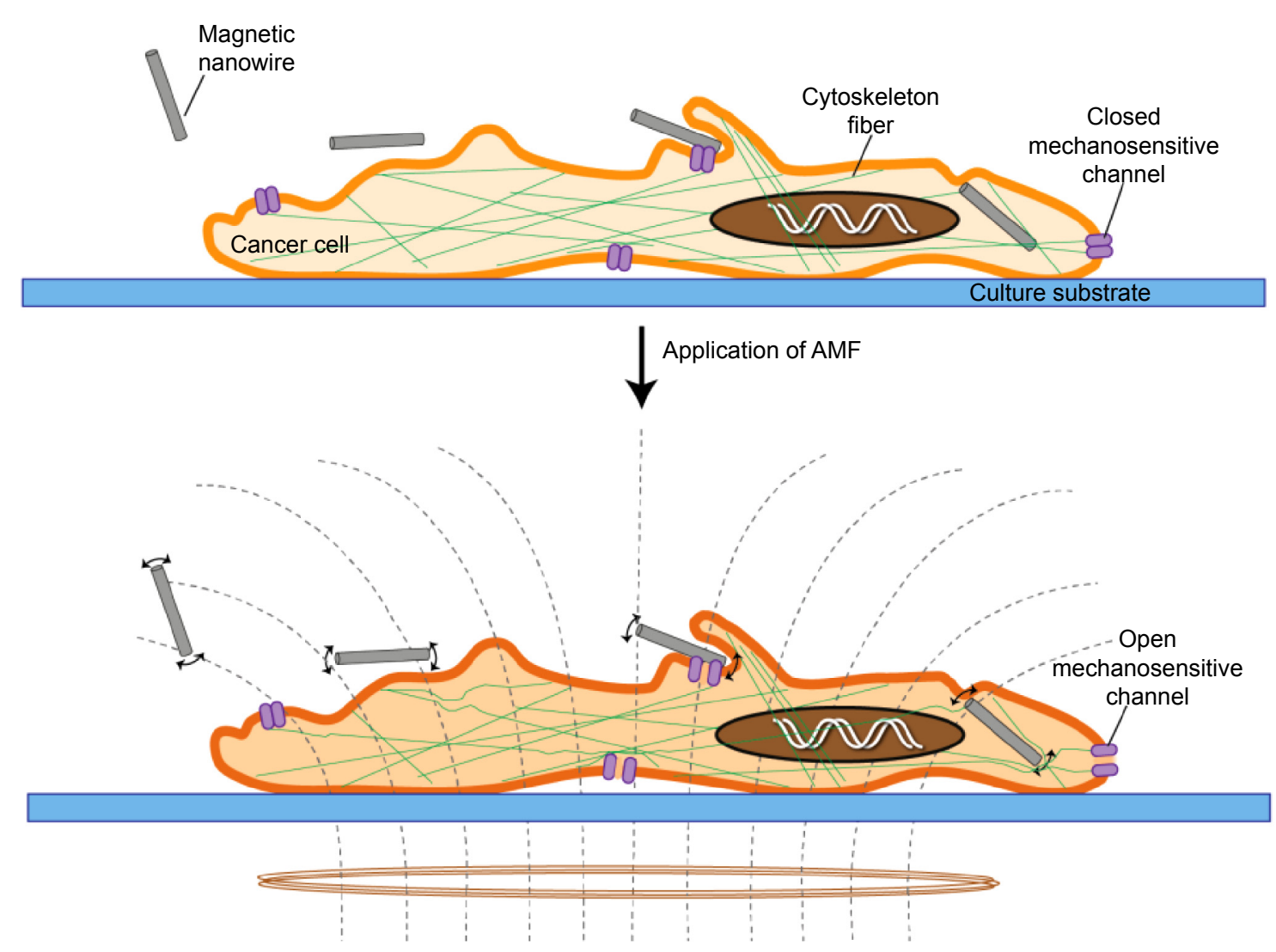

Figure 9 Schematic of the possible mechanism of cell death due to interaction of cancer cells with NWs and the subsequent application of a low-frequency, low-amplitude magnetic field.

Notes: The cytoskeleton fibers reorganize upon application of the magnetic field (evidence of F-actin reorganization due to magnetic field exposure is presented in Zablotskii et al ${ }^{37}$ ).

Abbreviations: AMF, alternating magnetic field; NWs, nanowires.

\section{Conclusion}

The interactions between cultured cancer cells, magnetic NWs, and AMFs were studied. The results showed that $\mathrm{Ni}$ NWs are quickly internalized (within less than 1 hour) by cultured colon cancer cells and do not cause extensive cytotoxicity at a concentration of $2.4 \mu \mathrm{g} / \mathrm{mL}$ and an incubation time of 1 hour, which confirms previous results obtained for incubation times up to 72 hours. ${ }^{33}$ The highest and significant viability drop of $11 \%$ was found for a higher NW concentration $(12 \mu \mathrm{g} / \mathrm{mL})$ and 1 hour incubation.

Exposing the cells to AMFs of $1 \mathrm{kHz}$ for 30 minutes led to small drops (maximum 5\%) in cell viability. Under the other AMF conditions tested, the cell viability values decreased slightly.

However, when an AMF is applied to cells that have internalized NWs, the cytotoxicity is strongly modulated, depending on the AMF frequency and NW concentration. The most efficient combination was observed at a NW concentration of $12 \mu \mathrm{g} / \mathrm{mL}$, where the cell viability dropped by $38 \%$ in case of a $1 \mathrm{kHz}$ field. For the $2.4 \mu \mathrm{g} / \mathrm{mL}$ concentration, the cell viability reduction was around $24 \%$ at both frequencies. Moreover, there was no relevant difference when applying the treatment for 10 minutes or 30 minutes. This evidence suggests that cell death induction mechanisms affect cells quickly, and treatment over an extended period of time does not add any benefits. Cell viability reduction was accompanied by cell membrane leakage, which is not a desirable process if the intended cell death pathway is apoptosis. Theoretically, leakage should not be caused by a single NW, because the force it exerts on the cell membrane is too small to rupture it. Rather, it seems to be the result of the forces generated by several NWs or agglomerated NWs.

While many underlying details of the mechanisms induced by magnetic NWs on cells under AMFs have yet to be elucidated, this study shows a possible approach to developing a non-chemotoxic or radiotoxic method to treat cancer cells. Figure 9 shows a schematic of the possible cell features accompanying cell death induction. An intriguing aspect of the method is the low power of the applied magnetic 
field. Not only does this low-power magnetic field have less chance of developing negative side effects, but treatment with a low-power magnetic field can be realized with compact and inexpensive equipment.

To take full advantage of this promising method, more fundamental studies as well as enhancements are required. For instance, cancer-cell specificity studies (using peptides or antibodies) and dispersion improvement studies to ensure homogeneous NW distribution within a cell population are needed. Regarding the latter, an issue observed throughout the experiments that could have a strong influence on the results is aggregation of NWs. Currently, protocols are being adapted to coat the NWs with polymers ${ }^{20}$ to improve their dispersion. Fundamental studies characterizing the particular type of cell death and its distinctive features as well as analyzing the molecular-biology mechanisms that can be triggered by the treatment, such as $\mathrm{Ca}^{2+}$ signaling and overexpression of proinflammatory cytokines, would be very useful.

\section{Acknowledgment}

Research reported in this publication was supported by the King Abdullah University of Science and Technology (KAUST).

\section{Disclosure}

The authors report no conflicts of interest in this work.

\section{References}

1. Pankhurst QA, Connolly J, Jones SK, Dobson J. Applications of magnetic nanoparticles in biomedicine. J Phys D Appl Phys. 2003; 36(13):R167-R181

2. Creixell M, Herrera AP, Ayala V, et al. Preparation of epidermal growth factor (EGF) conjugated iron oxide nanoparticles and their internalization into colon cancer cells. J Magn Magn Mater. 2010; 322(15):2244-2250.

3. Domenech M, Marrero-Berrios I, Torres-Lugo M, Rinaldi C. Lysosomal membrane permeabilization by targeted magnetic nanoparticles in alternating magnetic fields. ACS Nano. 2013;7(6):5091-5101.

4. McBain SC, Yiu HH, Dobson J. Magnetic nanoparticles for gene and drug delivery. Int J Nanomedicine. 2008;3(2):169-180.

5. Ruiz-Hernández E, Baeza A, Vallet-Regí M. Smart drug delivery through DNA/magnetic nanoparticle gates. ACS Nano. 2011;5(2): $1259-1266$.

6. Kubo T, Sugita T, Shimose S, Nitta Y, Ikuta Y, Murakami T. Targeted delivery of anticancer drugs with intravenously administered magnetic liposomes in osteosarcoma-bearing hamsters. Int J Oncol. 2000;17(2): 309-315.

7. Leung KC, Wang YX, Wang H, Xuan S, Chak CP, Cheng CH. Biological and magnetic contrast evaluation of shape-selective $\mathrm{Mn}-\mathrm{Fe}$ nanowires. IEEE Trans Nanobioscience. 2009;8(2):192-198.

8. Reich DH, Tanase M, Hultgren A, Bauer LA, Chen CS, Meyer GJ. Biological applications of multifunctional magnetic nanowires (invited). J Appl Phys. 2003;93(10):7275.

9. Gao J, Gu H, Xu B. Multifunctional magnetic nanoparticles: design, synthesis, and biomedical applications. Acc Chem Res. 2009;42(8): 1097-1107.
10. McCarthy JR, Weissleder R. Multifunctional magnetic nanoparticles for targeted imaging and therapy. Adv Drug Deliv Rev. 2008;60(11): $1241-1251$.

11. Gooneratne CP, Yassine O, Giouroudi I, Kosel J. Selective Manipulation of Superparamagnetic Beads by a Magnetic Microchip. IEEE Trans Magn. 2013;49:3418-3421.

12. Gooneratne CP, Giouroudi I, Liang C, Kosel J. A giant magnetoresistance ring-sensor based microsystem for magnetic bead manipulation and detection. J Appl Phys. 2011;109(7):07E517.

13. Li F, Kosel J. An efficient biosensor made of an electromagnetic trap and a magneto-resistive sensor. Biosens Bioelectron. 2014;59:145-150.

14. Li F, Kosel J. A Magnetic Method to Concentrate and Trap Biological Targets. IEEE Trans Magn. 2012;48(11):2854-2856.

15. Wang D, He J, Rosenzweig N, Rosenzweig Z. Superparamagnetic $\mathrm{Fe}_{2} \mathrm{O}_{3}$ Beads - CdSe/ZnS Quantum Dots Core-Shell Nanocomposite Particles for Cell Separation. Nano Lett. 2004;4(3):409-413.

16. Gao N, Wang H, Yang EH. An experimental study on ferromagnetic nickel nanowires functionalized with antibodies for cell separation. Nanotechnology. 2010;21(10):105107.

17. Choi D, Fung A, Moon H, et al. Transport of living cells with magnetically assembled nanowires. Biomed Microdevices. 2007;9(2):143-148.

18. Lin WS, Lin HM, Chen HH, Hwu YK, Chiou YJ. Shape effects of iron nanowires on hyperthermia treatment. J Nanomater. 2013:1-6.

19. Keshoju K, Xing H, Sun L. Magnetic field driven nanowire rotation in suspension. Appl Phys Lett. 2007;91(12):123114.

20. Günther A, Bender P, Tschöpe A, Birringer R. Rotational diffusion of magnetic nickel nanorods in colloidal dispersions. J Phys Condens Matter. 2011;23(32):325103.

21. Hultgren A, Tanase M, Chen CS, Meyer GJ, Reich DH. Cell manipulation using magnetic nanowires. J Appl Phys. 2003;93(10):7554-7557.

22. Hultgren A, Tanase M, Felton EJ, et al. Optimization of yield in magnetic cell separations using nickel nanowires of different lengths. Biotechnol Prog. 2005;21(2):509-515.

23. Ferré R, Ounadjela K, George JM, Piraux L, Dubois S. Magnetization processes in nickel and cobalt electrodeposited nanowires. Physical Review B. 1997;56(21):66-75.

24. Hultgren A, Tanase M, Chen CS, Reich DH. High-Yield Cell Separations Using Magnetic Nanowires. IEEE Trans Magn. 2004;40(4): 2988-2990.

25. Gao N, Yang X, Tsai Y-T, Chu GM, Wang H, Yang E-H. Antibody-functionalized magnetic nanowires for cell purification. George T, Islam MS, Dutta AK, editors. Micro-Nanotechnol Sensors, Syst Appl. 2009; 7318.

26. Zhao $\mathrm{Y}$, Zeng $\mathrm{H}$. Rotational maneuver of ferromagnetic nanowires for cell manipulation. IEEE Trans Nanobioscience. 2009;8(3):226-236.

27. Zhang L, Petit T, Peyer KE, Nelson BJ. Targeted cargo delivery using a rotating nickel nanowire. Nanomedicine. 2012;8(7):1074-1080.

28. Choi DS, Park J, Kim S, et al. Hyperthermia with magnetic nanowires for inactivating living cells. J Nanosci Nanotechnol. 2008;8(5): 2323-2327.

29. Choi DS, Hopkins X, Kringel R, Park J, Jeon IT, Keun Kim Y. Magnetically driven spinning nanowires as effective materials for eradicating living cells. J Appl Phys. 2012;111(7):07B329.

30. Hossain MZ, Kleve MG. Nickel nanowires induced and reactive oxygen species mediated apoptosis in human pancreatic adenocarcinoma cells. Int J Nanomedicine. 2011;6:1475-1485.

31. Kasprzak KS, Sunderman FW Jr, Salnikow K. Nickel carcinogenesis. Mutat Res. 2003;533(1-2):67-97.

32. Song MM, Song WJ, Bi H, et al. Cytotoxicity and cellular uptake of iron nanowires. Biomaterials. 2010;31(7):1509-1517.

33. Perez J, Contreras MF, Vilanova E, Ravasi T, Kosel J. Cytotoxicity and Effects on Cell Viability of Nickel Nanowires. In: E Purshotaman, editor. 2013 International Conference on Biological, Medical and Chemical Engineering. Hong Kong: DEStech Publications, Inc.; 2013: 178-184.

34. Prina-Mello A, Diao Z, Coey JM. Internalization of ferromagnetic nanowires by different living cells. J Nanobiotechnology. 2006;4:9. 
35. Poland C, Byrne F, Cho WS, et al. Length-dependent pathogenic effects of nickel nanowires in the lungs and the peritoneal cavity. Nanotoxicology. 2012;6:899-911.

36. Byrne F, Prina-Mello A, Whelan A, et al. High content analysis of the biocompatibility of nickel nanowires. J Magn Magn Mater. 2009; 321(10):1341-1345

37. Zablotskii V, Lunov O, Novotna B, et al. Down-regulation of adipogenesis of mesenchymal stem cells by oscillating high-gradient magnetic fields and mechanical vibration. Appl Phys Lett. 2014;105:103702.

38. Marcos-Campos I, Asín L, Torres TE, et al. Cell death induced by the application of alternating magnetic fields to nanoparticle-loaded dendritic cells. Nanotechnology. 2011;22(20):205101.

39. Kim DH, Rozhkova EA, Ulasov IV, et al. Biofunctionalized magneticvortex microdiscs for targeted cancer-cell destruction. Nat Mater. 2010;9:165-171.

40. Fung AO, Kapadia V, Pierstorff E, Ho D, Chen Y. Induction of Cell Death by Magnetic Actuation of Nickel Nanowires Internalized by Fibroblasts. The Journal of Physical Chemistry C. 2008;112: 15085-15088

41. Schmalz G, Schuster U, Schweik1 H. Influence of metals on IL-6 release in vitro. Biomaterials. 1998;19(18):1689-1694.

42. Garner LA. Contact dermatitis to metals. Dermatol Ther. 2004;17: 321-327.

43. Masuda H, Fukuda K. Ordered metal nanohole arrays made by a twostep replication of honeycomb structures of anodic alumina. Science. 1995;268(5216):1466-1468.

44. Nielsch K, Müller F, Li A-P, Gösele U. Uniform Nickel Deposition into Ordered Alumina Pores by Pulsed Electrodeposition. Adv Mater. 2000;12(8):582-586.

45. Pirota KR, Navas D, Hérnandez-Vélez M, Nielsch K, Vázquez M. Novel magnetic materials prepared by electrodeposition techniques: arrays of nanowires and multi-layered microwires. J Alloys Compd. 2004; 369(1-2):18-26.

46. Mosmann T. Rapid colorimetric assay for cellular growth and survival: application to proliferation and cytotoxicity assays. J Immunol Methods. 1983;65:55-63.

47. Kremb S, Helfer M, Heller W, et al. EASY-HIT: HIV full-replication technology for broad discovery of multiple classes of HIV inhibitors. Antimicrob Agents Chemother. 2010;54(12):5257-5268.

48. Karnosky M. Use of ferrocyanide-reduced osmium tetroxide in electron microscopy. Proceedings of the 11th Annual Meeting. American Society for Cell Biology; New Orleans, Louisiana; 1971;146.

49. Nielsch K, Wehrspohn RB, Barthel J, et al. Hexagonally ordered $100 \mathrm{~nm}$ period nickel nanowire arrays. Appl Phys Lett. 2001;79(9):1360.

50. Vega V, Böhnert T, Martens S, et al. Tuning the magnetic anisotropy of Co-Ni nanowires: comparison between single nanowires and nanowire arrays in hard-anodic aluminum oxide membranes. Nanotechnology. 2012;23(46):465709.

51. Zhang LW, Monteiro-Riviere NA. Mechanisms of quantum dot nanoparticle cellular uptake. Toxicol Sci. 2009;110(1):138-155.
52. Bridot J-L. Synthesis and characterization of iron oxide nanoparticles for cellular imaging Non specific cell labelling. Available from: http:// www.encite.org/html/img/pool/Bridot_ENCITE_Mons.pdf. Accessed October, 2014.

53. Chithrani BD, Ghazani AA, Chan WC. Determining the size and shape dependence of gold nanoparticle uptake into mammalian cells. Nano Lett. 2006;6(4):662-668.

54. Fröhlich E. The role of surface charge in cellular uptake and cytotoxicity of medical nanoparticles. Int J Nanomedicine. 2012;7:5577-5591.

55. Papageorgiou I, Brown C, Schins R, et al. The effect of nano- and micron-sized particles of cobalt-chromium alloy on human fibroblasts in vitro. Biomaterials. 2007;28(19):2946-2958.

56. Bakhru SH, Altiok E, Highley C, et al. Enhanced cellular uptake and long-term retention of chitosan-modified iron-oxide nanoparticles for MRI-based cell tracking. Int J Nanomedicine. 2012;7:4613-4623.

57. Lacava LM, Garcia VAP, Kückelhaus S, et al. Long-term retention of dextran-coated magnetite nanoparticles in the liver and spleen. J Magn Magn Mater. 2004;272-276:2434-2435.

58. Fojt L, Strasák L, Vetterl V, Smarda J. Comparison of the low-frequency magnetic field effects on bacteria Escherichia coli, Leclercia adecarboxylata and Staphylococcus aureus. Bioelectrochemistry. 2004;63(1-2): 337-341.

59. Strasák L, Vetterl V, Smarda J. Effects of low-frequency magnetic fields on bacteria Escherichia coli. Bioelectrochemistry. 2002;55(1-2): 161-164.

60. Aarholt E, Flinn EA, Smith CW. Effects of low-frequency magnetic fields on bacterial growth rate. Phys Med Biol. 1981;26(4):613-621.

61. Greene JJ, Skowronski WJ, Mullins JM, Nardone RM, Penafiel M, Meister R. Delineation of electric and magnetic filed of extremely low frequency electromagnetic radiation on transcription. Biochem Biophys Res Commun. 1991;174(2):742-749.

62. Wolf FI, Torsello A, Tedesco B, et al. 50-Hz extremely low frequency electromagnetic fields enhance cell proliferation and DNA damage: possible involvement of a redox mechanism. Biochim Biophys Acta. 2005; 1743(1-2):120-129.

63. Afrin R, Yamada T, Ikai A. Analysis of force curves obtained on the live cell membrane using chemically modified AFM probes. Ultramicroscopy. 2004;100(3-4):187-195.

64. Pierres A, Monnet-Corti V, Benoliel AM, Bongrand P. Do membrane undulations help cells probe the world? Trends Cell Biol. 2009;19(9): 428-433.

65. del Rio A, Perez-Jimenez R, Liu R, Roca-Cusachs P, Fernandez JM, Sheetz MP. Stretching Single Talin Rod Molecules Activates Vinculin Binding. Science. 2009;323:638-641.

66. Alon R, Dustin ML. Force as a facilitator of integrin conformational changes during leukocyte arrest on blood vessels and antigen-presenting cells. Immunity. 2007;26(1):17-27.

67. Watanabe I, Okada S. Effects of temperature on growth rate of cultured mammalian cells (L5178Y). J Cell Biol. 1967;32:309-323. 


\section{Supplementary materials}

Table SI Ni NW concentrations expressed in equivalent units

NW concentrations

\begin{tabular}{ll}
\hline Low & High \\
\hline $2.4 \mu \mathrm{g} / \mathrm{mL}$ & $12 \mu \mathrm{g} / \mathrm{mL}$ \\
$100 \mathrm{NW} / \mathrm{cell}$ & $500 \mathrm{NW} / \mathrm{cell}$ \\
$7.1 \times 10^{7} \mathrm{NW} / \mathrm{mL}$ & $3.5 \times 10^{8} \mathrm{NW} / \mathrm{mL}$
\end{tabular}

Abbreviation: NW, nanowire.

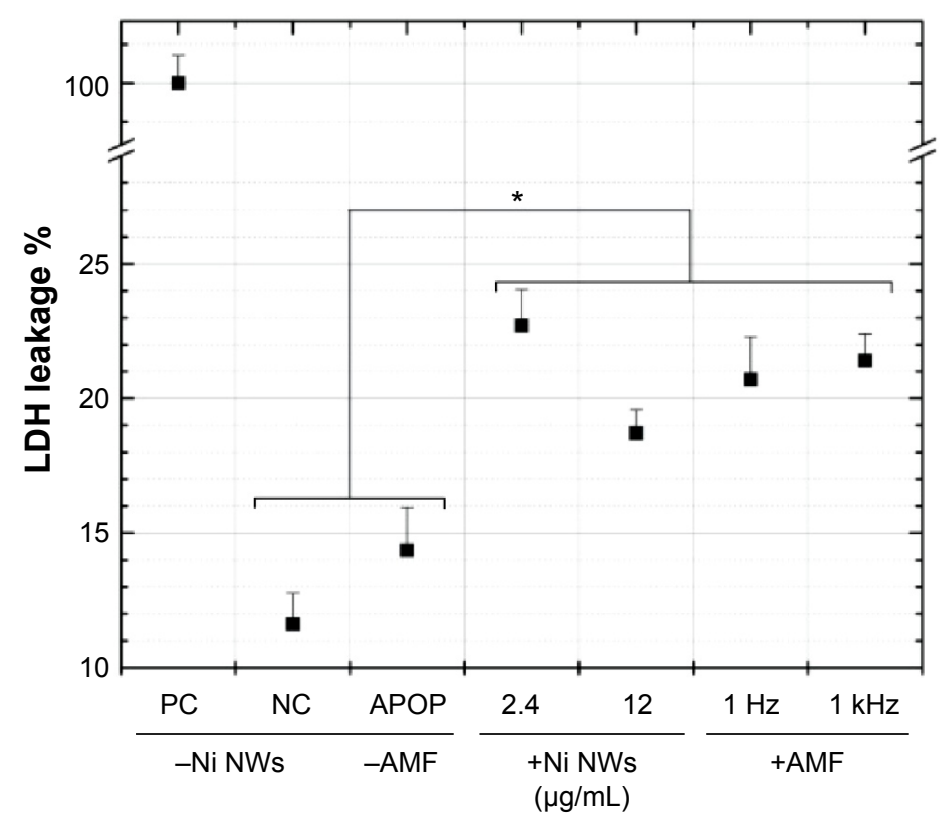

Figure SI LDH leakage of colon cancer cells under different conditions.

Notes: A sample of lysed cells was used as the PC which corresponded to 100\% leakage. No NWs were added to the NC cells. Apoptosis (APOP) was induced in cells by exposing them to ultraviolet radiation for I hour. Cells were incubated with NWs at two given concentrations for I hour (no AMF applied). Cells were exposed to AMF for 10 minutes (no NWs added). Data represent means \pm standard deviation, $n=3, * P<0.05$ versus NC/APOP. $P<0.0$ I for $P C$ versus all the other samples (data not shown). Abbreviations: AMF, alternating magnetic field; LDH, lactate dehydrogenase; NC, negative control; NWs, nanowires; PC, positive control.

\section{Publish your work in this journal}

The International Journal of Nanomedicine is an international, peerreviewed journal focusing on the application of nanotechnology in diagnostics, therapeutics, and drug delivery systems throughout the biomedical field. This journal is indexed on PubMed Central, MedLine, CAS, SciSearch ${ }^{\circledR}$, Current Contents ${ }^{\circledR} /$ Clinical Medicine,
Journal Citation Reports/Science Edition, EMBase, Scopus and the Elsevier Bibliographic databases. The manuscript management system is completely online and includes a very quick and fair peer-review system, which is all easy to use. Visit http://www.dovepress.com/ testimonials.php to read real quotes from published authors.

\footnotetext{
Submit your manuscript here: http://www.dovepress.com/international-journal-of-nanomedicine-journal
} 